\title{
DETERMINAÇÃO DO NÚMERO ADEQUADO DE REPETIÇÕES PARA A AVALIAÇÃO DA VIRADA NO NADO LIVRE
}

MS. GRAZIELA AVELINE SILVEIRA

Mestre em Ciências do Movimento Humano pela UDESC

Atua na área da Biomecânica - (Santa Catarina - Brasil)

e-mail: grazisilveira@hotmail.com

\section{MS. GUSTAVO RICARDO SCHÜTZ}

Mestre em Ciências do Movimento Humano pela UDESC

Professor Substituto da UDESC. Atua na área da Biomecânica - (Santa Catarina - Brasil) e-mail: gugaschutz@hotmail.com

\section{MS. LUCIANA GASSENFERTH ARAUJO}

Mestre em Ciências do Movimento Humano pela UDESC

Atua na área da Biomecânica - (Santa Catarina - Brasil)

e-mail: lugassen@yahoo.com.br

\section{MS. ELINAI DOS SANTOS FREITAS}

Mestre em Ciências do Movimento Humano pela UDESC

Professora da UNISUL. Atua na área da Biomecânica (Santa Catarina - Brasil)

e-mail: elinaifreitas@hotmail.com

\section{GABRIEL FERNANDES JACOMEL}

Graduando em Educação Física pela UDESC,

Atua na área da Biomecânica (Santa Catarina - Brasil)

e-mail: gabrielfernandesjacomel@yahoo.com.br

\section{DR. HELIO ROESLER}

Doutor em Engenharia Mecânica pela UFRGS e

Professor do Depto. de Ciências da Saúde da UDESC (Santa Catarina - Brasil)

e-mail:d2hr@udesc.br 


\section{RESUMO}

O estudo propõe um procedimento para coletas de dados da virada no nado livre (número de execuções). Onze nadadores executaram 8 viradas cada um. Analisou-se as variáveis Pico de Força normalizado (PFn), Tempo de Contato (TC), Impulso (Imp) e Tempo de Virada em 10 metros (TVIOm). A estabilidade visual quanto ao número de execuções foi verificada pelo cálculo do coeficiente de variação acumulado que apresentou curvas com variações discretas. Foi verificado se ocorria diferença entre as médias acumuladas usando a análise de variância (ANOVA) entre os diferentes agrupamentos. Não houve diferenças significativas. Para confirmar o número mínimo de repetições, confrontou-se os dados pelo teste de $U$ de Mann-Whitney. Não houve diferenças significativas. Observou-se que três execuções válidas são o suficiente para avaliar o desempenho na virada.

PALAVRAS-CHAVE: Nado livre; virada; biomecânica.

\section{INTRODUÇÃO}

A natação é um esporte em constante aprimoramento, seja pela técnica biomecânica dos estilos ou pelos recursos utilizados. A luta contra o tempo em competições de alto nível impulsionou os estudos, por parte de técnicos e pesquisadores, para o desenvolvimento de métodos mais eficazes de treinamento e precisão dos movimentos, buscando o melhor desempenho dos nadadores (ARAUJO et al., 2001 ; MARTINS, 200 I). Isto significa que o nadador precisa não somente nadar rápido, mas também executar rapidamente e com perfeição as saídas e viradas se houver (GUIMARÃES E HAY, 1985).

A fim de identificar meios mais eficientes de superar as adversidades impostas pelo meio líquido, profissionais da área têm recorrido aos recursos biomecânicos. Em se tratando de biomecânica na natação, as viradas são os gestos mais estudados e por isso constituem um tema de muita discussão, pois são decisivas em provas (MAGLISCHO, 1999; SÁNCHES, 2000), e, portanto, fundamentais para o resultado final dos atletas. Segundo Maglischo ( 1999), em uma prova de 50 m nado livre em piscina curta o tempo de virada pode atingir entre $20 \%$ e $38 \%$ do tempo total da prova. A otimização deste gesto vem sendo avaliada por alguns pesquisadores (BLANKSBY et. al, 1996; LYTTLE \& MASON, 1997; DANIEL et. al, 2003; MAANON et. al, 2003; PRINS \& PATZ, 2006; PEREIRA et. al, 2006).

Com o decorrer de novos testes, através de avaliações biomecânicas, verificou-se a necessidade de padronizar as metodologias de avaliação, bem como protocolos. Hubert (2005) ressalta a aplicação de critérios e protocolos para análises de viradas, uma vez que os atletas tendem há esperar muito tempo durante as coletas. 
Apesar da realização de vários estudos com viradas na natação, muitos parâmetros ainda estão sem uma padronização ou protocolos definidos, como o número de execuções a serem avaliadas. Como exemplos desta discrepância entre autores, Martins (200 I) avaliou nove viradas de cinco sujeitos especialistas em provas de nado livre; Hubert et al. (2003) avaliaram doze viradas de apenas um nadador tentando detectar a influência das variáveis dinâmicas no tempo de execução da virada no nado livre; Para Araújo (2004) oito viradas de 38 sujeitos foram necessárias para avaliar a técnica de virada no nado livre e Blanksby et al. (1996) verificaram três execuções para analisar a virada no nado livre de um grupo de 36 nadadores.

Dentro deste contexto, este estudo objetivou, a partir de indicadores biomecânicos de desempenho, propor o número de execuções necessárias para realização de coletas de dados na virada no nado livre.

\section{MATERIAL E MÉTODOS}

Participaram do estudo I I nadadores de ambos os sexos, escolhidos de maneira intencional, tendo domínio da técnica de execução da virada com rolamento no nado livre, com média de idade de $16 \pm 3$ anos, média de massa corporal de $63,3 \pm 8,9 \mathrm{~kg}$, média de estatura 172,6 \pm 8,0 m. $\bigcirc$ estudo foi aprovado pelo Comitê de Ética em Pesquisa em Seres Humanos da Universidade do Estado de Santa Catarina, sob o número de referência 52/2007.

Entre os indicadores biomecânicos do desempenho da virada optou-se pelas variáveis dinamométricas: Pico de Força (PFn): maior valor registrado da força aplicada pelo nadador na parede/plataforma de força durante a execução da virada, sendo o valor adquirido normalizado pelo peso corporal [BW]; Tempo de Contato (TC): tempo em que o nadador mantém contato com a parede/ plataforma de força, obtido pela subtração do tempo final $\left(t_{f}\right)$ de contato dos pés do tempo inicial de contato $\left(\mathrm{t}_{\mathrm{i}}\right)$ [s]; Impulso (Imp): integral de uma força (força aplicada perpendicular à plataforma) durante o intervalo de tempo em que ela atua (tempo em que o nadador permanece em contato com a plataforma) sendo determinado através da integração numérica da curva Força normalizada $\times$ TC, [N.s/N] (ARAUJO, 2004). Optou-se por estas variáveis por estarem relacionadas com o desempenho da virada (TAKAHASHI et al., 1984; BLANKSBY et al., 1999; HUBERT, 2003; LYTTLE, 1999; ARAUJO, 2006; SHAHBAZI et al., 2007).

Para aquisição dos dados dinamométricos foi utilizada uma plataforma de força subaquática extensométrica (ROESLER, 1997) (FIGURA I - "a") e também um sistema formado por placa condicionadora de sinas, conversor A/D e computador 
(Figura I - "b"), desenvolvidos com base nos estudos de Roesler (1997; 2003). O software de aquisição de dados utilizado foi o SAD 32 Bits (SILVA \& ZARO, 1997). A frequência de aquisição foi de $600 \mathrm{~Hz}$.

A plataforma de força foi acoplada a um suporte para sua fixação dentro da piscina, no plano vertical, encostada na parede de virada da raia 3. O suporte tem a finalidade de sustentar a plataforma e possui um formato em "L", com as dimensões de 0,70 m por 0,54 m. A altura da plataforma com o suporte é de 0,20 m, ficando a tampa da plataforma afastada 0,20 $\mathrm{m}$ da parede da piscina. Para que a faixa preta do fundo da piscina fique na distância oficial da parede da piscina (plataforma de força) foi feita uma adaptação recuando-a 0,20 m (Figura I - "c").

Uma moldura nas dimensões 2,24 × 1,22 × 0,20 m (largura $\times$ altura $\times$ espessura) foi acoplada a parede da piscina, envolvendo a plataforma de força. Esta moldura tem a finalidade de "mascarar" a plataforma fornecendo aos nadadores segurança e a sensação de estarem tocando a parede da piscina normalmente (Figura I - "d").

Os dados dinamométricos foram tratados no próprio SAD 32 Bits, a partir da seguinte rotina: ( I ) aplicação do coeficiente de calibração e filtragem (filtro passa baixa FFT tipo Butterworth, frequência de corte $30 \mathrm{~Hz}$ ); (2) normalização pelo peso corporal; (3) verificação do PFn; (4) verificação do TC; (5) verificação do Imp.

Para aquisição da variável cinemática tempo de virada em 10 m - TVIOm (5,0 × 5,0 m), foi utilizada uma câmera de vídeo, posicionada a 5,2 m da borda e a 5,0 m da plataforma, (Figura I - "e"). A câmera estava perpendicular a piscina e como marcador para a distância de 5,0 m, foi utilizada uma corda de nylon (Figura I - "f") em toda extensão da piscina, perpendicularmente à orientação das raias. $\bigcirc$ desempenho foi determinado pelo tempo decorrido desde $\mathrm{o}$ instante em que a cabeça do nadador atinge a marca da distância referida em direção a borda da piscina $\left(\mathrm{t}_{\mathrm{i}}\right)$, executa a virada e retorna a marca $\left(\mathrm{t}_{\mathrm{f}}\right)$, obtido por meio da contagem dos quadros a partir do $\left(\mathrm{t}_{\mathrm{i}}\right)$ até $\mathrm{o}\left(\mathrm{t}_{\mathrm{f}}\right)$, multiplicado pelo valor I/30 (frequência de aquisição de imagens de $30 \mathrm{~Hz}$; resolução de $0,033 \ldots$ s), [s]. A contagem de quadros foi realizada no software InterVideo, Inc. WinProducer, versão 3. 


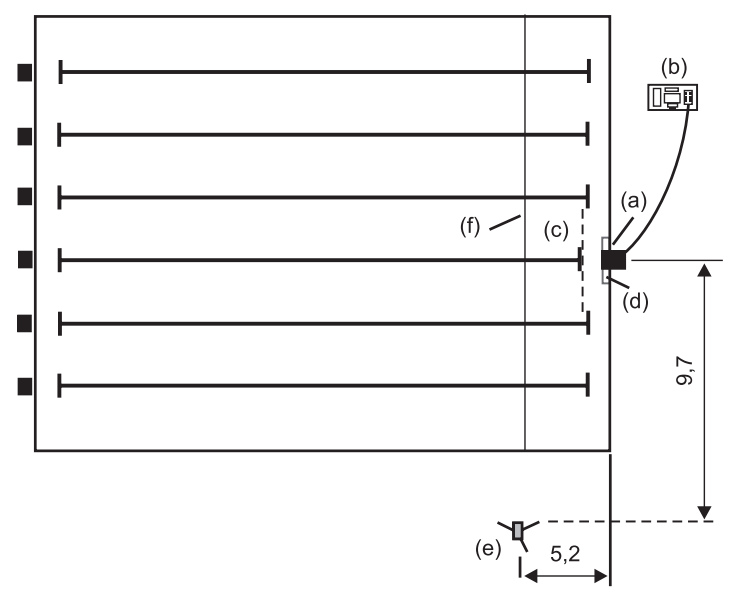

Figura I. Layout da piscina para aquisição de dados: a) plataforma de força subaquática; sistema de aquisição de dados; c) "maquiagem" no fundo da piscina; d) moldura; e) câmera dos 5,0 m; f) corda de nylon.

A coleta de dados foi realizada nas dependências da piscina do CEFID/ UDESC, previamente preparada, sendo a sessão de treinamento programada pelo técnico da equipe, procurando familiarizar os nadadores com a situação de coleta de dados e equipamentos, tendo as seguintes ações: a) orientação dos procedimentos da coleta e assinatura do Termo de Consentimento Livre e Esclarecido e Consentimento para Fotografias, Vídeo e Gravações; b) mensuração das características antropométricas massa e estatura; c) realização do aquecimento pré-estabelecido e algumas execuções de virada sobre a plataforma de força para adaptação ao instrumento; d) execução de oito viradas para cada nadador sequencialmente, com intervalo de 5 minutos entre cada execução. As saídas foram realizadas de dentro da piscina (parede de saída), onde os nadadores deveriam atingir a velocidade máxima nos $12,5 \mathrm{~m}$, realizavam a virada e mantinham a velocidade máxima até os $12,5 \mathrm{~m}$, diminuindo até chegar ao local de partida.

Para a caracterização dos dados foi utilizada a estatística descritiva, com a identificação dos valores de média $(\bar{x})$, desvio padrão (s) e coeficiente de variação (CV). A normalidade dos dados foi realizada através do teste de Shapiro-Wilk $(\alpha=0,05)$.

Diferentes opções estatísticas foram realizadas a fim de determinar o número ideal de repetições, sendo: I) verificação da estabilidade visual através do cálculo do coeficiente de variação acumulado; 2) verificação da diferença entre as médias acumuladas por meio da análise de variância (ANOVA) entre os diferentes agrupamentos (grupo com I execução $X$ grupo com 2 execuções $X$ grupo com 
3... X grupo com 8 execuções); 3) confrontar os dados, por meio do teste de $U$ de Mann-Whitney, realizado entre as variáveis nos seguintes arranjos: comparar as execuções impares e pares ( $1,3,5$, e $7 \times 2,4,6$ e 8$)$, entre as quatro primeiras e as quatro últimas execuções ( I , 2, 3 e $4 \times 5,6,7$ e 8), e entre as três primeiras e as três seguintes ( I , 2 e $3 \times 4,5$ e 6); 4). Utilizou-se para o processamento estatístico o pacote estatístico SPSS for Windows I I.0, a um nível de significância de 5\%.

\section{RESULTADOS E DISCUSSÃO}

Na Tabela I são descritos os resultados dos indicadores biomecânicos de desempenho da virada.

Tabela I. Valores das variáveis PFn, TC, Imp e TVIOm ( $\mathrm{n}=88)$.

\begin{tabular}{lllll}
\hline & $\mathrm{PFn}[\mathrm{N} / \mathrm{N}]$ & $\mathrm{TC}[\mathrm{s}]$ & $\mathrm{Imp}[\mathrm{N} . \mathrm{s} / \mathrm{N}]$ & $\mathrm{TVIOm}[\mathrm{s}]$ \\
\hline $\bar{X}$ & 1,72 & 0,32 & 0,34 & 5,52 \\
$\mathrm{~s}$ & 0,35 & 0,08 & 0,05 & 0,57 \\
$\mathrm{CV}$ & $20,3 \%$ & $25,0 \%$ & $14,7 \%$ & $10,3 \%$ \\
\hline
\end{tabular}

$(\bar{X})$ = média, (s) = desvio padrão, (CV\%) = coeficiente de variação

Os dados obtidos neste estudo para o PFn são semelhantes aos encontrados por Hubert et al. (2002), que observaram valores entre de I,7 a 2,5 N/N, e por Roesler (2003), que apresentou valores médios de I,9 N/N. Em relação ao TC, observou-se um valor médio de 0,32 s entre os nadadores, estando de acordo com estudo de Lyttle e Mason ( 1997) que encontraram a mesma média (0,32 s). O valor médio encontrado para o Imp foi de 0,34 N.s/N, sendo semelhante aos achados de Roesler (2003), que verificou média de 0,37 N.s/N, e de Hubert et al. (2003), que observaram valores médios de 0,36 N.s/N. É importante ressaltar que os estudos citados compararam nadadores com características semelhantes em relação ao desempenho. A variável TVIOm registrou média próxima a de Lyttle et al. (1997) que obtiveram a média de tempo de 5,07s.

Neste estudo optou-se em avaliar oito viradas no nado livre, tentando determinar um número mínimo para pesquisas posteriores. A definição de um número mínimo de viradas é importante para aperfeiçoar o processo de coleta e análise de dados (HUBERT, 2005). Outros motivos podem ser citados: em processos longos 
de avaliação os resultados podem ser influenciados pelo efeito da aprendizagem do gesto a ser realizado, além de se tornarem desmotivantes o que poderia interferir no desempenho, como cita Figueiredo (2000) em seu estudo.

A partir dos valores obtidos, a estabilidade visual quanto ao número de execuções foi verificada através do cálculo do coeficiente de variação acumulado (MELO, 1995), indicando o número de tentativas necessário para aceitar os dados medidos pelo instrumento/método. Mead e Curnow (1986) corroboram com esta ideia, citando o coeficiente de variação como uma medida importante sobre a variabilidade dos resultados podendo ser útil na definição do número de repetições de ensaios. $\mathrm{Na}$ Figura 2 é apresentado o coeficiente de variação acumulado para cada variável.

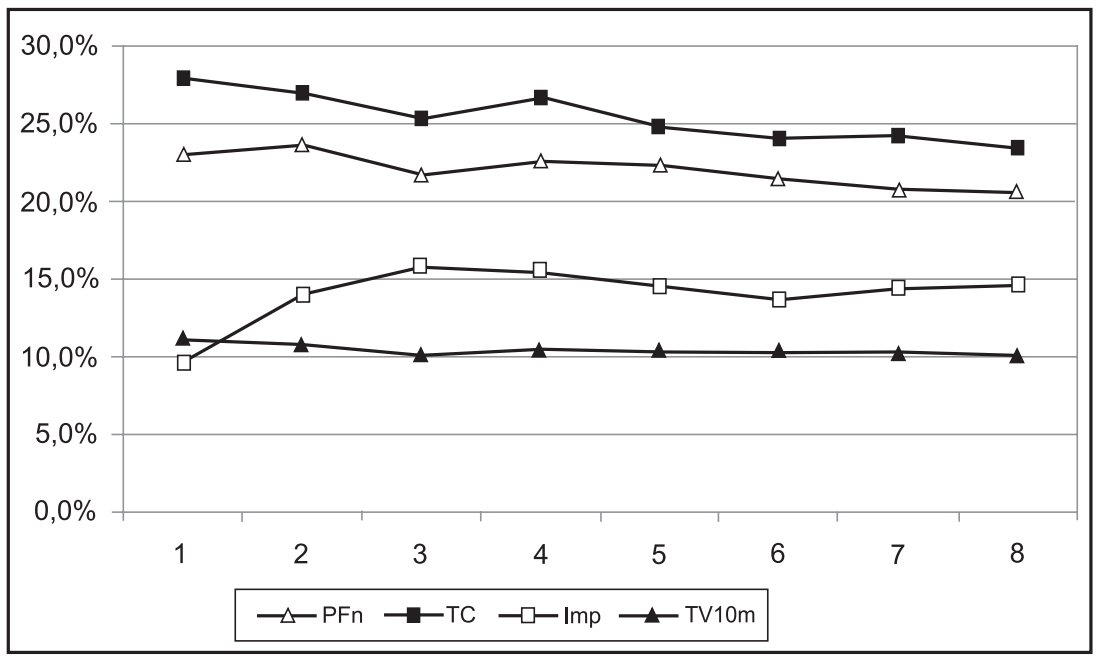

Figura 2. Coeficiente de variação acumulado das varáveis PFn, TC, Imp e TVIOm.

A determinação visual do ponto de estabilização da curva do coeficiente de variação experimental é um método subjetivo e, como tal, sujeito à variação entre observadores (SILVA et al., 2003). Buscou-se então determinar visualmente, o ponto o qual a estimativa dos parâmetros torna-se estável. Entretanto, o comportamento das curvas do coeficiente de variação acumulado apresentou variações discretas dificultando as afirmações a respeito. Somente na variável Imp pode-se verificar uma discrepância inicial seguida de uma estabilização a partir da terceira execução. Os valores encontrados se enquadram aos esperados, como cita Gill (1987), que o pesquisador deve estar atento sobre os valores considerados razoáveis do $C V$ em sua área de pesquisa. 
Segundo este, coeficientes de variação menores que $1 \%$ são raros em Ciências Biológicas, o que talvez não o seja nas Ciências Físicas, e muitas características biológicas apresentam coeficientes de variação na faixa entre 5 e 50\%.

A variabilidade entre as repetições pode gerar um erro e quanto maior for essa variabilidade, maior será o coeficiente de variação, menor a precisão e maior o número de repetições necessárias para representar determinado caráter (GOMES, 1990). Entretanto, observou-se no gráfico uma tendência a estabilização das curvas a partir da terceira repetição.

Uma segunda possibilidade foi verificar se ocorria diferença entre as médias acumuladas. Utilizando-se do método proposto por Hubert (2004), realizou-se a análise de variância (ANOVA) entre os diferentes agrupamentos. Não foram encontradas diferenças significativas $(p>0,05)$ entre as médias acumuladas para todas as variáveis.

Outra opção foi confrontar os dados em diversos arranjos por meio do teste de $U$ de Mann-Whitney, mas também não foram verificadas diferenças significativas $(p>0,05)$.

Os indicadores biomecânicos de desempenho estão relacionados ao tempo em que se executa a tarefa, sendo medido pelo tempo gasto pelo nadador para percorrer uma determinada distância (MAGLISCHO, 1999; NAVARRO, 1995; HALJAND, 1998). Observou-se um aumento crescente na média acumulada da variável temporal TVI 0 m à medida que era exigido um maior número de repetições, como apresentado na Figura 3.

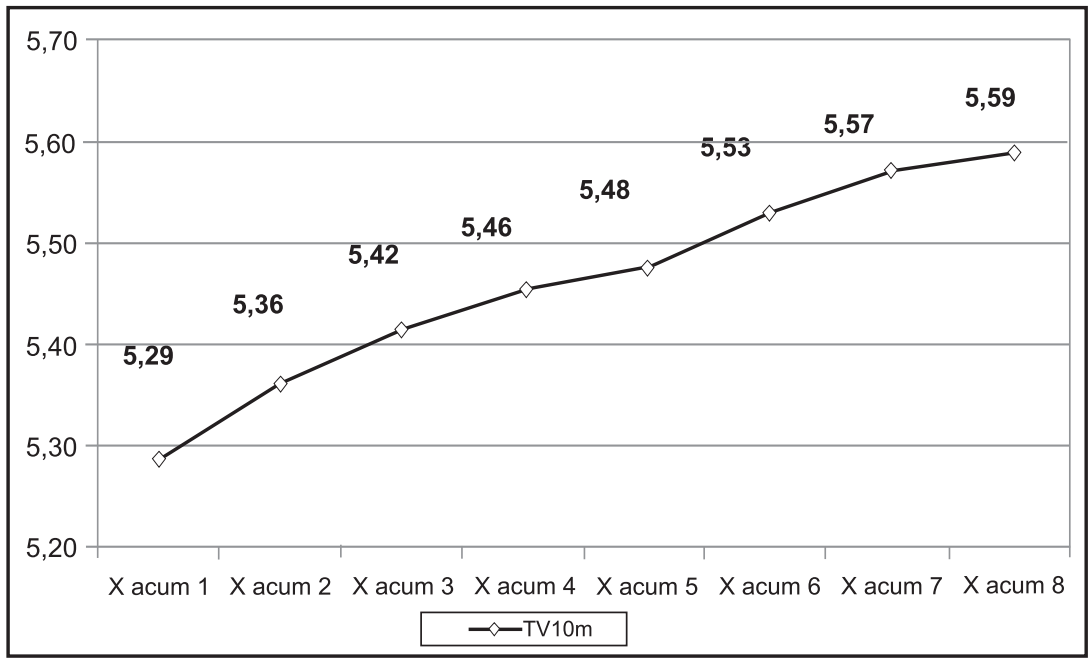

Figura 3. Média acumulada para TVIOm e TVI5m com relação ao número de repetições. 
Tratando-se de natação, onde centésimos de segundo decidem vencedores (PEREIRA, 2006), o aumento do TVI Om observado resulta na queda do desempenho, e um número exagerado de execuções não seria adequado. Outro ponto a ser observado é o comportamento dos nadadores, pois em certos momentos durante as coletas, mostravam-se irritados, podendo interferir na execução da sua virada.

Sugere-se então, a utilização de três execuções válidas para a análise de variáveis dinamométricas e cinemáticas, como já observados nos trabalhos de Blanksby et al. ( 1 996), Lyttle et al. (1999), Daniel et al. (2003) e Roesler (2003), mesmo sem estes salientarem o porquê desta escolha.

Entretanto o pesquisador pode achar prudente realizar um número maior de execuções, pois pode ocorrer alguma falha não detectada durante a coleta, ocorrendo perdas, caracterizando a execução como inválida. O pesquisador pode então, aumentar o número de execuções visando uma margem de segurança para a coleta.

\section{CONCLUSÃO}

Com relação ao número mínimo de execuções necessárias para avaliar o desempenho na virada, se observou uma tendência que três execuções válidas sejam o suficiente. Entretanto, um número maior de execuções pode ser utilizado como segurança, em caso de algum imprevisto não detectado no momento da coleta de dados.

A padronização das coletas facilita o procedimento reduzindo o tempo e os erros da pesquisa. Isto permite um aperfeiçoamento dos estudos sobre a virada na natação, e assim, viabilizar a pesquisadores a avaliação do desempenho de nadadores de diferentes níveis.

\section{Determination of appropriete number of repetition to evaluate the freestyle swimming turn}

ABSTRACT: The aim of this study was to develop a procedure of data collection on freestyle swimming turn (number of repetition). Eleven swimmers executed 8 turns each one. The measured variables were Normalized Peak Force (PFn), Contact Time (TC), Impulse (Imp) and Turn Time in 10 meters (TVIOM). The visual stability as for the number of executions was checked through the calculation of the accumulate coefficient of variation that presents small variations curves. No significant differences were found between the accumulate averages using ANOVA between the different groupings and also for the confirmation of the least number of repetitions, it chose in confronting the data, through the Mann-Whitney $U$ test carried out between the variables. Was found that three valid executions are enough to evaluate the performance in front freestyle turn.

KEYWORDS: Freestyle; swim turn; biomechanics. 
Determinación del número adecuado de repeticiones para la evaluación del volteo en el nado libre

RESUMEN: El estudio propone un procedimiento para colectas de datos del volteo en el nado libre (número de ejecuciones). Once nadadores ejecutaron 8 volteos cada uno. Se analizo las variables de Pico de Fuerza normalizado (PFn), Tiempo de Contacto (TC), Impulso (Imp) e Tiempo de Viragem en 10 metros (TVIOm). La estabilidad visual cuanto al número de ejecuciones fue verificad por el cálculo del coeficiente de variable acumulado que presento curvas con variaciones discretas. Fue verificado si ocurrían diferencias entre las medidas acumuladas usando el análisis de variabilidad (ANOVA) entre los diferentes agrupamientos. No hubo diferencias significativas. Para confirmar el número mínimo de repeticiones se compararon los datos de $U$ de Mann-Whitney. No hubo diferencias significativas. Se observo que tres ejecuciones válidas son suficientes para evaluar el desempeño en el volteo.

PALABRAS CLAVE: Nado libre; volteo; biomecánica.

\section{REFERÊNCIAS}

ARAUJO, L. G. Análise biomecânica da virada no nado Crawl. 2004. 55 f. Dissertação (Mestrado em Ciências do Movimento Humano) - Centro de Ciências da Saúde e do Esporte, Universidade do Estado de Santa Catarina, Florianópolis, 2004.

ARAUJO, L. G.; ROESLER, H.; PEREIRA, S. M.; MARTINS, E. R. S.; PEREIRA, B. N. Análise dinamométrica da virada com rolamento do nado crawl. In: CONGRESSO BRASILEIRO DE BIOMECÂNICA, 9., 200I, Gramado. Anais... Porto Alegre: UFRGS, 200I . p. 80-84.

ARAUJO, L. G.; ROESLER, H.; PEREIRA, S. M.; MARTINS, E. R. S.; PEREIRA, B. N. Análise dinamométrica da virada com rolamento do nado crawl. Anais do $9^{\circ}$ Congresso Brasileiro de Biomecânica, 200I, Gramado, Brasil. Porto Alegre: UFRGS; 2001.2 p.80 - 4.

BLANKSBY, B. A. Gaining on turns. Proceedings of XVII International Symposium On Biomechanics In Sports, Perth, p. 82-85, 1999.

BLANKSBY, B. A.; GATHERCOLE D. G.; MARSHALL R. N. Force plate and video analysis of the tumble turn by age-group swimmers. The Journal of Swimming Research, Fort Lauderdale, v. II, n. I, p. 40-45, fall, 1996.

DANIEL, K.; KLAUCK, J. \& BIEDER, A. Kinematic and Dynamographic Research in Different Swimming Turns. Biomechanics and Medicine in Swimming IX, Saint-Etienne, p. 20 I-206, 2003.

FIGUEIREDO, S. H. Variáveis que interferem no desempenho do atleta de alto rendimento. In RUBIO, K. (Org). Psicologia do Esporte: Interfaces, pesquisa e Intervenção. São Paulo: Casa do Psicólogo. p. I 13- 124. 2000.

GILL, J. L. Design and analysis of experiments in the animal and medical sciences. Ames: The lowa State University Press. lowa, 1987. 
GOMES, F. P. Curso de estatística experimental. 13. ed. Piracicaba: São Paulo: Nobel, 1990.

GUIMARÃES, A. C. S.; HAY, J. G. A mechanical analisis of the grab starting technique in swimming. International Journal of Sport Biomechanics, Newark, vol. I, n. I, p. 25-35, fev. 1985.

HALJAND, R. Roles of Starts and Turns at Short Course Events. In: L. E. d. Natation (Ed.). Sheffield: Ponds Forge International Sports Center, 1998.

HUBERT, M.; ROESLER, H.; ARAÚJO, L.; G.; PEREIRA, S. M.; SANTOS, L. M.; SCHUTZ, G. R. Influência de Variáveis Dinâmicas no Tempo de Execução da Virada no Nado Crawl. In: CONGRESSO BRASILEIRO DE BIOMECÂNICA, 2003, Ouro Preto. Anais do $10^{\circ}$ Congresso Brasileiro de Biomecânica, 2003, v. I, p. 207-210.

HUBERT, M.; SCHUTZ, G. R.; CARDOSO, T. R.; ARAÚJO, L. G.; ROESLER, H. Relação entre o pico máximo de força e o tempo de execução da virada com rolamento do nado crawl. Revista Brasileira de Medicina do Esporte, Niterói, v. 8, n. 5, p. 197-197, Set/Out, 2002.

HUBERT, M.; SCHUTZ, G. R.; SILVEIRA, G. A.; RUSCHEL, C.; ROESLER, H. Comportamento de variáveis biomecânicas da saída na natação: comparação de diferentes técnicas e nados. Lecturas Educación Física y Deportes. Buenos Aires, v. 10, 2006. Disponível em: <http://www.efdeportes.com/efd9l/>. Acesso em: jun. 2008.

LYTTLE, A.; MASON, B. A Kinematic and kinetic analysis of the freestyle and butterfly turns. The Journal of Swimming Research, Fort Lauderdale, v. 12, n. I, p. 7- I I, fall, 1997.

MAAÑON, R.; SÁNCHEZ, J.; EIROA, J.; BRAÑA, S.; MON, J. Evolution of the Crawl Turn After Technical Intervention in the Swimming Training. Biomechanics and Medicine in Swimming IX. Saint-Etienne, p. 20I-206, 2003.

MAGLISCHO, E. Nadando ainda mais rápido. Rio de Janeiro: Guanabara, 1999.

MARTINS, E. R. S. Análise dinâmica da virada com rolamento no nado Crawl, 200 I . 74 f. Dissertação (Mestrado em Ciências do Movimento Humano) - Programa de Pós-Graduação em Ciências do Movimento Humano, Universidade do Estado de Santa Catarina, Florianópolis, 200 I.

MEAD, R.; CURNOW, R. N. Statistical methods in agriculture and experimental biology. New York: Chapman and Hall, 1986.

MELO, S. I. L. Um sistema para determinação do coeficiente de atrito entre calçados esportivos e pisos usando o plano inclinado. 1995. 221 f. Tese (Doutorado em Ciências do Movimento Humano) - Programa de Pós-Graduação em Ciências do Movimento Humano, Universidade Federal de Santa Maria, Santa Maria, 1995.

NAVARRO, F. Hacia el dominio de la natacion. Madrid: Gimnos, 1995.

PEREIRA, S. M.; ARAUJO, L. G.; FREITAS, E. S.; GATTI, R. G.; SILVEIRA, G. A. Biomechanical analysis of the turn in front crawl swimming. Biomechanics and Medicine in Swimming $X$, Porto, v. 6, p. 77-79, 2006. 
PRINS, J. H.; PATZ, A. The influence of tuck index, depth of foot-plant, and wall contact time on the velocity of push-off in the freestyle flip turn. Biomechanics and Medicine in Swimming X. Porto, v. 6, p. 82-85, 2006.

ROESLER, H. Desenvolvimento de plataforma subaquática para medições de forças e momentos nos três eixos coordenados para a utilização em Biomecânica. 1997. 193p. Tese (Doutorado em Engenharia Mecânica) - Programa de Pós-Graduação em Engenharia Mecânica, Universidade Federal do Rio Grande do Sul, Porto Alegre, 1997.

ROESLER, $H$. Turning Force Measurement in Swimming Using Underwater Force Platform. Biomechanics and Medicine in Swimming IX. Saint-Etienne, p. 243-248, 2003.

SÁNCHEZ, J. A. Estudio sobre recursos metodológicos no usuales en el proceso de enseñanza-aprendizaje del viraje estilo crol. In: Congreso Internacional de Actividades Acuáticas y Natación Deportiva, 2000, Toledo. Anais do XX Congreso Internacional de Actividades Acuáticas y Natación Deportiva, 2000.

SHAHBAZI, M. M.; SANDERS, R. H.; MCCABE, C.; ADAMS, D. Investigating Correlations Between Swim Pike Turn Kinematics Variables in Front crawl. Proc. of 25 th International Symposium on Biomechanics in Sports, 2007. Ouro Preto, p. 381-384, 2007.

SILVA, L.; ZARO, A. SAD VERSÃO 3.2 - "Sistema de Aquisição de dados - Manual de Operação". Caderno técnico de Engenharia Mecânica CT07, DEMEC. Porto Alegre: UFRGS, 1997.

SILVA, R. L.; XAVIER, A.; LEITE, H. G.; PIRES, I. E. Determinação do tamanho ótimo da parcela experimental pelos métodos da máxima curvatura modificado, do coeficiente de correlação intraclasse e da análise visual em testes clonais de eucalipto. Revista Árvore, Viçosa, v. 27, n. 5, p. 669-976, set./out. 2003.

TAKAHASHI, G.; YOSHIBA, A.; TSUBAKIMOTO, S. Propulsive force Generated by Swimmers during a Turning Motion. In: INTERNATIONAL SYMPOSIUM OF BIOMECHANICS IN SWIMMING, 4, 1982. Proceedings of the... Champaign: Human Kinetic Publishers, 1984. p. 192-198.

Recebido: 08 jun. 2009

Aprovado: 14 set. 2010

Endereço para correspondência: Gabriel Fernandes Jacomel

Rua Pascoal Simone, 358, Laboratório de Pesquisas em Biomecânica Aquática Bairro Coqueiros Florianópolis - SC CEP: 88.080-350 